\title{
Encore is a member of a novel family of proteins and affects multiple
}

\section{processes in Drosophila oogenesis}

\author{
Cheryl Van Buskirk1, Nancy C. Hawkins ${ }^{2}$ and Trudi Schüpbach ${ }^{1, *}$ \\ ${ }^{1}$ Howard Hughes Medical Institute, Department of Molecular Biology, Princeton University, Princeton NJ 08544, USA \\ 2Department of Molecular and Cell Biology, University of California at Berkeley, Berkeley CA 94720, USA \\ *Author for correspondence (e-mail: gschupbach@molbio.princeton.edu)
}

Accepted 30 August; published on WWW 24 October 2000

\section{SUMMARY}

Mutations in the encore (enc) gene of Drosophila melanogaster cause one extra round of mitosis in the germline, resulting in the formation of egg chambers with extra nurse cells. In addition, enc mutations affect the accumulation of Gurken protein within the oocyte, leading to the production of ventralized eggs. Here we show that enc mutants also exhibit abnormalities in karyosome morphology, similar to other ventralizing mutants such as okra and spindle $B$. Unlike these mutants, however, the defects in Gurken accumulation and karyosome formation do not result from activation of a meiotic checkpoint.
Furthermore, we demonstrate that the requirement for enc in these processes is temporally distinct from its role in germline mitosis. Cloning of the enc locus and generation of anti-Enc antibodies reveal that enc encodes a large novel protein that accumulates within the oocyte cytoplasm and colocalizes with grk mRNA. We argue that the enc mutant phenotypes reflect a role for Enc in the regulation of several RNA targets.

Key words: Drosophila, encore, gurken, oskar, Karyosome, Oogenesis

\section{INTRODUCTION}

Drosophila oogenesis can be used as a system in which to analyze the control of cell division because the germline mitoses that give rise to the egg chamber follow a highly regulated, stereotyped pattern (for a review of oogenesis, see Spradling, 1993). Egg production begins in a region of the ovary called the germarium, where germline stem cells divide asymmetrically to produce cystoblasts. Each cystoblast undergoes precisely four rounds of mitosis, with incomplete cytokinesis, to give rise to a cluster of sixteen cells interconnected by ring canals. Two cells within the cyst possess four ring canals, and one of these cells will become the oocyte. The cyst becomes enveloped by a layer of somatically derived follicle cells, and the newly formed egg chamber exits the germarium and moves down the ovariole as it develops into an egg. Within the oocyte nucleus, the chromatin condenses into a hollow sphere known as the karyosome as part of progression through prophase I of meiosis. The other fifteen germline cells undergo endoreplication and differentiate as polyploid nurse cells.

The polarity of the egg is established by communication between the oocyte and the surrounding follicle cells (Schüpbach, 1987). This communication is mediated by the EGF receptor (EGFR) signaling pathway (for review, see Van Buskirk and Schüpbach, 1999). EGFR is expressed throughout the follicular epithelium (Kammermeyer and Wadsworth, 1987; Sapir et al., 1998), and localized activation of this receptor at one end of the egg chamber establishes posterior follicle cell fates. Later in oogenesis, the receptor is activated on one side of the egg chamber to establish dorsal follicle cell fates. As the pattern of cell fates within the follicular epithelium determines the polarity of the egg shell and the embryo, the initial events that determine follicle cell fate are critical. Both of these germline-to-soma signaling events are mediated by the same ligand, the TGF $\alpha$-like molecule gurken (grk) (Neuman-Silberberg and Schüpbach, 1993; GonzálezReyes et al., 1995; Roth et al., 1995). The production and localization of this ligand within the oocyte are tightly regulated, and several mutations have been isolated that affect patterning of the egg through an effect on grk regulation (for review, see Nilson and Schüpbach, 1999).

Mutations in encore (enc) disrupt the regulation of the germline divisions that give rise to the 16-cell cyst, as well as the establishment of the axes of the egg (Hawkins et al., 1996, 1997). Two P-element insertion mutations and fourteen EMSinduced enc alleles display similar defects. enc mutant egg chambers undergo an extra round of mitosis in the germline, giving rise to egg chambers with 31 nurse cells and an oocyte. This mitotic defect is temperature sensitive, being highly penetrant in most alleles at $25^{\circ} \mathrm{C}$. One possible target of enc function in cystocyte division is the gene bag of marbles (bam) (McKearin and Spradling, 1990). In wild-type germaria, bam RNA expression is restricted to cystoblasts and two-cell cysts, whereas in enc mutant germaria, the domain of bam RNA expression is expanded to include more cystocytes. Genetic evidence suggests that this expansion contributes to the enc extra division, as mutations in bam dominantly suppress the 
enc mitotic defect (Hawkins et al., 1996). When enc mutant females are raised at $18^{\circ} \mathrm{C}$, the mitotic defect is rare. However, under these conditions the eggs that are produced have fused or absent dorsal appendages, structures that are normally secreted by dorsal follicle cells. The reduction of these dorsal structures is reminiscent of the ventralized eggs produced by females mutant for $g r k$ or Egfr, and in fact it has been shown that Grk protein levels are reduced in enc mutant egg chambers (Hawkins et al., 1997). The levels of grk RNA appear unaffected, however, suggesting that enc plays a role in the translation or stability of Grk protein within the oocyte.

Here we show a previously undetected defect in karyosome formation in oocytes of enc mutant females. Several other mutations have been identified which, like enc, affect both the formation of the karyosome and the accumulation of Grk within the oocyte (González-Reyes et al., 1997; Ghabrial et al., 1998). Some of these, such as okra and spindle B (spn-B), have been shown to be involved in the repair of double-stranded breaks induced during meiotic recombination. In these mutants, the presence of unrepaired recombination intermediates causes activation of a meiotic checkpoint, leading to defects in karyosome formation and, surprisingly, a reduction in the accumulation of Grk (Ghabrial and Schüpbach, 1999). These effects appear to be mediated through modification of Vasa protein, a potential translational regulator of grk (Styhler et al., 1998, Tomancak et al., 1998) in response to activation of the checkpoint. Here we have used genetic and molecular analysis to show that enc affects karyosome formation and Grk expression independently of the meiotic checkpoint-vasa pathway, suggesting an additional level of regulation of these processes. We have cloned the enc locus and characterized Enc protein expression, and we find that Enc is a member of a novel class of proteins, conserved between flies and humans, that shows a dynamic localization within the developing oocyte.

\section{MATERIALS AND METHODS}

\section{Drosophila stocks}

Two P-element insertion mutations, enc $c^{A 309}$ and $e n c^{B B}$, as well as 14 EMS-induced enc alleles were described by Hawkins et al. (1996). All of these alleles display a temperature-sensitive extra round of germline mitosis as well as a cold-sensitve ventralized egg phenotype. The excision mutation enc ${ }^{R 17}$, derived from the A309 P-element insertion, was described by Hawkins et al. (1997) and is the only allele that shows no division defect despite severe DV patterning defects. Hemizygous $e n c^{R 17}$ females do, however, produce a low percentage of extra-division egg chambers. The mei-41D3 allele was kindly provided by Scott Hawley.

\section{Molecular cloning and rescue constructs}

Standard molecular techniques were carried out as described by Sambrook et al. (1989). Plasmid rescue from the BB129 P-element insertion was performed as described by Pirrotta (1986). Rapid amplification of cDNA ends was performed using a 5' RACE kit from Gibco with $1 \mu \mathrm{g}$ of total ovarian RNA, and enc-specific primers for first-strand synthesis (5'ACAATCACGGCTGGCGTCTC) and PCR amplification (5'GCCCTTCATGTTGCCTCGTC). Primer extension was performed as described by Kim et al. (1995) using a primer at the $5^{\prime}$ end of the sequence obtained from RACE (5'CCTTTGTGGCACGCATTTTCAATCGCGGCCC 3 '). For northern analysis, a $1 \mathrm{~kb}$ Sall-EcoRI genomic fragment near the $3^{\prime}$ end of the enc cDNA was used to detect the 7 and $8 \mathrm{~kb}$ transcripts. The probe that detected only the $8 \mathrm{~kb}$ transcript was a $750 \mathrm{bp}$ fragment extending from the EcoRI site to the end of the longer class of enc cDNAs. The transgenic rescue construct contained a $5.5 \mathrm{~kb}$ fragment (nt 570-6090) of the enc cDNA under the control of the hsp83 promoter in a pCasper transformation vector. Four insertion lines were obtained, with the highest expression level, as detected by antibody staining, from hspEnc5.5-14, inserted on the X chromosome, which was sufficient to rescue the enc mitotic defect. In order to rescue the cold-sensitive patterning defects of enc, a line carrying multiple copies of the transgene was constructed, with hspEnc5.5-14 on the X chromosome, hspEnc5.5-2 on the second chromosome, and the enc ${ }^{U U 3}$ mutation on the third. The GenBank accession number for the $6858 \mathrm{nt}$ enc cDNA sequence is AF243382, extending from the 5'-most RACE sequence to the $3^{\prime}$ end of the longer transcript class. The enc cDNA is contained within nucleotides $132,273-154,795$ of the 309,883 nt genomic scaffold corresponding to GenBank accession number AE003479.

The P-element associated with enc $^{A 309}$ is inserted at nt 132, 310 of this genomic sequence, and the enc ${ }^{r 17}$ excision extends upstream from this site into the neighboring $\operatorname{scs} \alpha$ gene (accession number AAF47796), though the $5^{\prime}$ limit of this deletion is yet to be determined. Another scs $\alpha$ homolog is present in the Drosophila genome (AAF55672).

\section{Production of anti-Enc antibodies}

Nucleotides 1079-1703 of the enc cDNA sequence, corresponding to amino acids 133-340, were amplified by PCR (Clonetech) adding a $5^{\prime}$ BamHI restriction site and $3^{\prime}$ Pst I and EcoRI sites. To generate a GST-Enc fusion, the product was subcloned into the BamHI-EcoRI sites of pGEX-2T (Pharmacia). To generate an N-terminally 6XHistagged Enc fusion, the BamHI-PstI fragment was cloned into pQE9 (Qiagen). The GST-Enc fusion protein was purified for use in production of rat polyclonal anti-Enc antibodies. The His-tagged fusion served as antigen in the production of rabbit polyclonal antibodies which were used in immunoprecipitation.

\section{Immunohistochemistry and OliGreen staining}

Ovaries were dissected in phosphate-buffered saline (PBS), fixed in $200 \mu \mathrm{l} \%$ paraformaldehyde in PBST (PBS $+0.2 \%$ Tween 20) plus $600 \mu \mathrm{l}$ heptane for 20 minutes. For staining with the DNA-binding dye OliGreen (Molecular Probes), ovaries were incubated in 0.2 $\mathrm{mg} / \mathrm{ml}$ RNase A plus a 1/5000 dilution of OliGreen for 1 hour. For antibody staining, ovaries were blocked in PBST $+1 \%$ BSA, incubated overnight at $4^{\circ} \mathrm{C}$ with primary antibody, washed, and incubated with fluorescent secondary antibody for 1 hour at room temperature. After several washes, ovaries were mounted in glycerol:PBS and visualized by confocal microscopy. Rat anti-Enc serum was used at a 1:1000 dilution and rabbit anti-Vasa, a gift from P. Lasko, was used at 1:1000. Rat anti-Osk serum, provided by A. Ephrussi, was used at 1:4000. All dilutions and washes were in PBST.

\section{Double labeling of ovaries for RNA and protein}

Ovaries were fixed as described above, but with the addition of $20 \mu \mathrm{l}$ of DMSO. Subsequent steps were carried out as described by Tautz and Pfeifle (1989) using a hybridization temperature of $55^{\circ} \mathrm{C}$, with the following modifications. The secondary antibody used to detect the $1.7 \mathrm{~kb}$ digoxigenin-labeled grk probe was an unconjugated antidigoxigenin antibody from sheep (Boehringer Mannheim), and was added along with rat anti-Enc primary antibody. A cy5-conjugated anti-sheep tertiary antibody was used for fluorescent detection of the RNA, and was added at the same time as the cy3-conjugated rat secondary antibody used to detect Enc.

\section{Enc Immunoprecipitation and western blots}

Protein A/G beads (Santa Cruz Biotechnology) were incubated with either pre-immune or anti-Enc serum from rabbits $(1 \mu \mathrm{l}$ raw serum per $\mu \mathrm{l}$ beads) for 30 minutes on ice. Lysates were prepared by 
dissecting 20 ovaries into cold PBS and grinding for 15 seconds in $100 \mu \mathrm{l}$ cold lysis buffer plus protease inhibitors $(50 \mathrm{mM}$ Tris $\mathrm{pH} 8$, $150 \mathrm{mM} \mathrm{NaCl}, 1 \mathrm{mM}$ EDTA, $1 \% \mathrm{NP}-40,2 \mu \mathrm{g} / \mathrm{ml}$ aprotinin, $1 \mu \mathrm{g} / \mathrm{ml}$ pepstatin, and $0.5 \mathrm{mg} / \mathrm{ml}$ AEBSF; 4-(2-aminoethyl)benzensulphonyl fluoride). The lysate was spun 5 minutes in a microcentrifuge at maximum speed, and $5 \mu \mathrm{l}$ of the supernatant was set aside for use as a whole cell lysate control. The remaining lysate was added to $20 \mu \mathrm{l}$ of pre-immune serum-coated beads. After a 30 minute incubation on ice, the beads were pelleted at low speed and the pre-cleared lysate was added to $20 \mu \mathrm{l}$ of the anti-Enc-coated beads. After incubation, beads were washed in lysis buffer, resuspended in an equal volume of protein gel loading buffer, boiled, and loaded onto a $7.5 \%$ polyacrylamide gel. Transfer to nitrocellulose (Amersham) was performed in standard transfer buffer with the addition of $0.1 \%$ SDS to aid in transfer of large proteins. Blots were blocked in TBST (Trisbuffered saline + Tween 20) $+5 \%$ milk, incubated overnight at $4^{\circ} \mathrm{C}$ with rat anti-Enc serum at 1:1000, washed and incubated with HRPconjugated anti-rat antibody (Amersham) at 1:2000. After several washes in TBST, bands were visualized using chemiluminescent detection (Amersham).

\section{RESULTS}

\section{Oocyte nuclear morphology in enc mutants}

Mutations in encore have been previously described to cause defects in both cystocyte division and in dorsoventral (DV) patterning of the egg (Hawkins et al., 1996, 1997). These phenotypes are separable based on their temperature sensitivity. Mutant females raised at $25^{\circ} \mathrm{C}$ produce egg chambers that have undergone one extra round of germline mitosis and from which eggs with wild-type DV pattern are produced. At $18^{\circ} \mathrm{C}$, the mitotic defect is rare but the eggs produced are ventralized, and this has been shown to be due to a decrease in the amount of Grk protein within the oocyte. In addition, at this temperature certain alleles of enc cause the production of egg chambers containing an oocyte with a partially polyploid 'nurse cell-like' nucleus, which may reflect a defect in oocyte differentiation (Hawkins et al., 1996). We have further examined the morphology of oocyte nuclei in enc mutant egg chambers, and we have observed additional abnormalities in nuclear morphology that are distinct from the previously described oocyte differentiation defect.

In wild-type egg chambers, the oocyte chromosomes condense early in oogenesis to form a compact structure, the karyosome, which persists until late oogenesis (reviewed by Spradling, 1993; Fig. 1A,B). In enc mutant egg chambers, however, in addition to the previously described polyploid oocyte nucleus phenotype (Fig. 1C), we find more subtle defects in the formation of the karyosome. In all alleles examined, a fraction of the egg chambers contain oocytes in which the karyosome has a less compact appearance (Fig. 1D). Often the nuclear material is split into two or three lobes (Fig. 1E,F) or can be seen to line the inside of the (E) $e n c^{R 17}$. (F) enc ${ }^{A 309}$. nuclear membrane (Fig. 1G). While it is possible that these karyosome defects are related to the polyploid oocyte nucleus phenotype, homozygous enc ${ }^{R 17}$ females, which show severe defects in karyosome formation, do not display the previously described oocyte differentiation defect, suggesting that these phenotypes represent perturbations of separate processes.

\section{enc does not act through the meiotic checkpoint}

Several genes have been described that cause a reduction in the amount of Grk protein within the oocyte and, in addition, show defects in the morphology of the oocyte nucleus similar to those seen in enc. Among these are vasa (Styhler et al., 1998; Tomancak et al., 1998), the spindle A-E genes (Gonzáles-Reyes et al., 1997), okra, aubergine, deadlock, squash and zucchini (Ghabrial et al., 1998). Recent characterization of okra and spn- $B$ has revealed an unexpected relationship between the meiotic cycle of the oocyte and the control of Grk translation (Ghabrial et al., 1998; Ghabrial and Schüpbach, 1999). These genes were found to encode proteins with homology to known DNA repair enzymes, and were shown to be required for the repair of recombination-induced double strand breaks during Drosophila meiosis. Both the karyosome defect and the ventralized egg phenotype produced by mutations in these genes were found to be suppressed by a null mutation in $\mathrm{mei}$ 41, a Drosophila homolog of MEC1, which is required to arrest the cell cycle in response to unrepaired double-strand breaks in S. cerevisiae (for review, see Roeder, 1997). Additionally, it was found that activation of the meiotic checkpoint leads to modification of Vasa (Vas), as detected by an aberrant electrophoretic migration of the protein in spn-B mutant extracts (Ghabrial and Schüpbach, 1999). vas encodes a DEAD-box containing RNA helicase with homology to eIF4A and is implicated in the translational regulation of oskar (osk) and grk (Lasko and Ashburner, 1988; Hay et al., 1988; Styhler et al., 1998; Tomancak et al., 1998). Consistent with vas acting downstream of the meiotic checkpoint, mutations in vas display karyosome and grk translation defects which are not suppressible by mutations in mei-41 (Ghabrial and Schüpbach, 1999).

In order to determine whether the abnormal karyosome morphology and decreased Gurken levels seen in enc mutants could be a secondary consequence of the activation of a meiotic
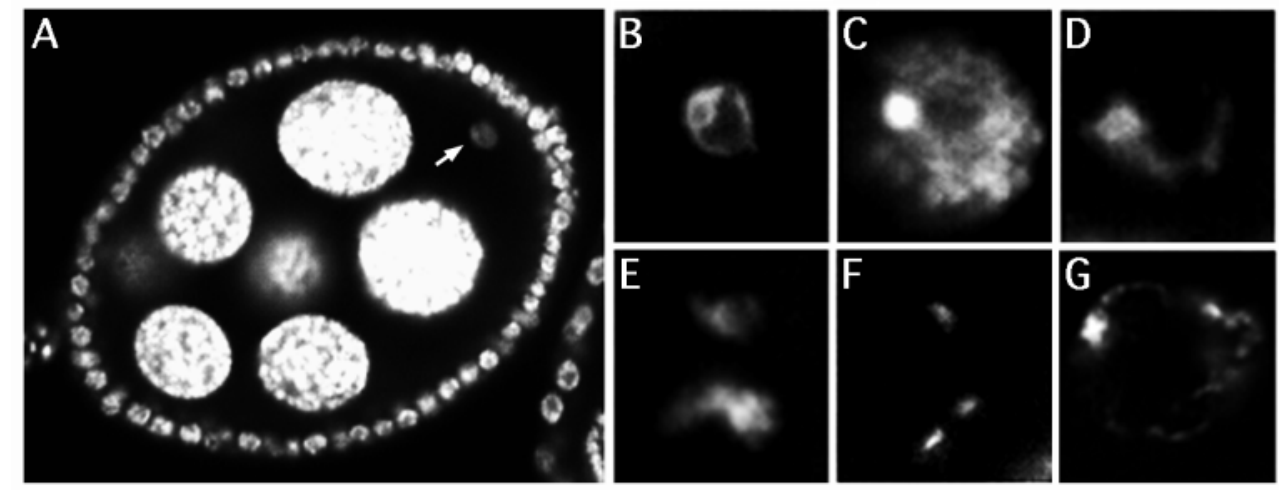

Fig. 1. enc mutant egg chambers show abnormal karyosome morphology. (A) A wild-type egg chamber stained with OliGreen, with the karyosome indicated (arrow), and magnified in B. (C-G) Homozygous enc mutant karyosomes, at same scale as B. (C,G) enc ${ }^{Q 4}$. (D) enc ${ }^{U U 3}$. 
Table 1. $m e i-41^{D 3}$ does not suppress the egg shell and karyosome defects of enc

\begin{tabular}{|c|c|c|c|c|}
\hline & $\mathrm{Eg}$ & 11 pher & e $(\%)$ & \\
\hline & I & II & III & karyosomes \\
\hline$m e i-41^{D 3} /+;$ enc $^{R 17} / \mathrm{Df}$ & 0 & 9.1 & 90.9 & 48.2 \\
\hline$m e i-41^{D 3} / m e i-41^{D 3} ;$ enc $^{R 17} / \mathrm{Df}$ & 0 & 10.2 & 89.8 & 45.1 \\
\hline
\end{tabular}

Classification of egg shell phenotype with respect to dorsoventral polarity was based on the appearance of the dorsal appendages. Class I: wild type, with two dorsal appendages. Class II: moderately ventralized, with a single dorsal appendage. Class III: strongly ventralized, with little or no dorsal appendage. Females were raised at $18^{\circ} \mathrm{C}$. Over 400 eggs were scored for each genotype.

checkpoint, we analyzed the phenotype of females mutant for both enc and mei-4l (Table 1). Our results show that neither the ventralization nor the karysome defects of enc are suppressed by $m e i-41^{D 3}$, a null allele that suppresses $o k r$ and spn-B mutants to the extent that mainly wild-type (Class I) eggs are produced (Ghabrial and Schüpbach, 1999). This result indicates that the role of enc in Grk regulation is not through an effect on meiotic progression, but rather that enc mutations behave like mutations in vas, which affect Grk accumulation and oocyte nuclear morphology but are not suppressed by loss of mei-4l.

We also examined whether the expression of Vas protein is affected in enc mutants, since modification of Vas appears to down-regulate its activity in okra and $s p n-B$ mutants. If enc were required to activate Vas activity in response to meiotic progression, we would expect the mobility of Vas in enc mutant extracts to be altered. However, we did not observe a shift in the electrophoretic migration of Vas in enc mutants, nor did the levels of Vas appear to be affected (data not shown), and hence Vas seems not to be a target of enc function. Enc therefore appears to affect karyosome formation and Grk protein accumulation independently of the meiotic checkpoint pathway and may act either in conjunction with or downstream of vas, or in a parallel pathway affecting these processes.

\section{oskar is translated ectopically within enc oocytes}

grk-Egfr signaling is required not only for the establishment of dorsal follicle cell fates, but also earlier in oogenesis for the determination of the posterior follicle cells (Roth et al., 1995; González-Reyes et al., 1995). A signal from these cells is in turn responsible for the polarization of the oocyte along the anterior-posterior (AP) axis. Thus in the absence of $g r k$ signaling early in oogenesis, the AP polarity of the oocyte is not correctly established, and oskar RNA, which is normally found at the posterior of the oocyte, becomes localized to the center (Roth et al., 1995; González-Reyes et al., 1995). Consistent with the defects in $g r k$ signaling in enc mutants, osk RNA is mislocalized in $40 \%$ of enc $c^{R 17} / \mathrm{Df}$ mutant egg chambers (Hawkins et al., 1997). We also wished to examine Osk protein expression in enc mutants, as certain genes such as aubergine (Wilson et al., 1996), which are implicated in Grk translation, are also required for Osk translation. In examining Osk protein expression in enc mutant egg chambers, we often found the protein localized normally at the posterior pole (Fig. 2A,B), and in some cases localized to
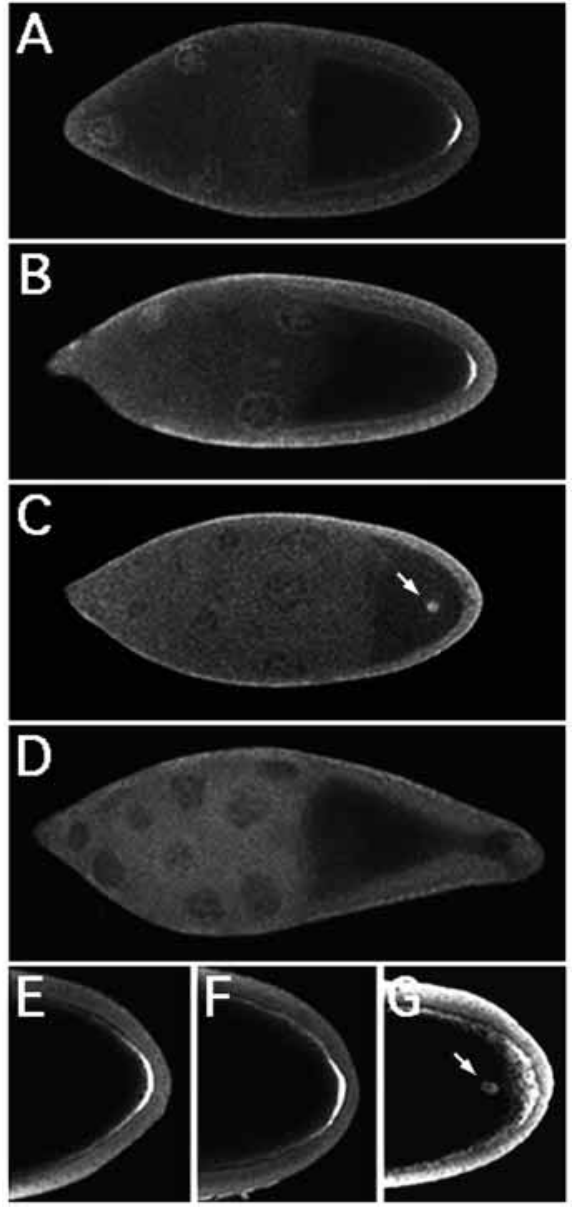

Fig. 2. Oskar and Vasa protein expression in enc mutant egg chambers. (A-D) Osk antibody stainings. (A) In wild type, Osk protein is detected only at the posterior pole of the oocyte. $(B, C)$ In enc ${ }^{R 17} / \mathrm{Df}$ mutant egg chambers, Osk can be found at the posterior and/or in the center of the oocyte (arrow). (D) In grk $^{2 B 6}$ mutant egg chambers, Osk protein is not detected. (E-G) Vasa protein expression. (E) Wild type. (F,G)enc $c^{R 17} / \mathrm{Df}$.

an internal site within the oocyte (Fig. 2C). In addition, we frequently observed egg chambers in which Osk could be found at both sites (not shown). Thus the distribution of Osk protein is similar to the pattern of osk RNA localization in these mutant egg chambers, and hence enc is not required for Osk translation.

We were surprised to find that even the mislocalized osk RNA was translated in enc, given that in $g r k^{2 B 6}$ mutants, in which we find osk RNA mislocalized in $99 \%$ of egg chambers, we never detect any Osk protein (Fig. 2D). The lack of Osk protein expression in grk mutants, and in other mutants with abolished posterior localization of Osk, has been shown previously (Markussen et al., 1995; Rongo et al., 1995), and thus it has been suggested that proper osk RNA localization is required for its translation. Recently, however, mutant situations have been found in which this is not the case. In egg chambers mutant for par-1, or those with Laminin A posterior follicle cell clones, osk RNA and protein can be found tightly localized to the center of the oocyte (Tomancak et al., 2000; Deng and Ruohola-Baker, 2000). Our 


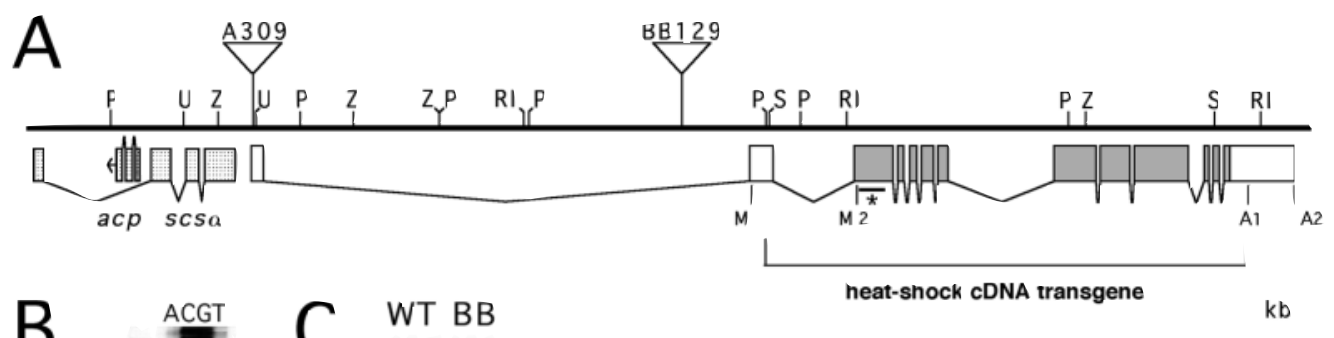

antibody epitope (*), and the $3^{\prime}$ ends of the two classes of enc transcripts (A1, A2) are indicated. Shaded boxes indicate the predicted open reading frame. $\mathrm{P}$ elements associated with the $e n c^{A 309}$ and $e n c^{B B}$ mutations are shown, and other transcripts in the region are indicated by stippled boxes. The transcript for succinyl-CoA synthetase alpha subunit $(\operatorname{scs} \alpha)$ lies just upstream of $e n c$, and is transcribed in the same direction. The gene encoding accesory gland protein in $63 \mathrm{~F}(a c p 63 F)$ is nested with an intron of $\operatorname{scs} \alpha$ and is transcribed in the opposite direction. The genomic region shown here coresponds to nt 127,800-155,000 of GenBank AA003479. (B) Primer extension from the 5'-most exon of enc, showing a strong reversetranscriptase stop (arrowhead) at the same site as the $5^{\prime}$ end of the enc cDNA sequence, and also showing another stop (arrow) 300-400 nt upstream of this, which may represent the $5^{\prime}$ end of the transcript. (C) Northern analysis of ovarian RNA from wild type and $e n c^{B B}$

homozygotes, using the $1 \mathrm{~kb}$ SalI-EcoRI fragment near the $3^{\prime}$ end of the enc cDNA as probe. Transcripts of $7 \mathrm{~kb}$ and $8 \mathrm{~kb}$ are detected in wild-type ovarian RNA which are absent from $e n c^{B B}$. Expression of $\operatorname{scs} \alpha$ was also examined and found to be unaffected in $e n c^{B B}$ (not shown). oskar (osk) transcript is used as a loading control. results with enc confirm that localization of osk RNA to the posterior of the oocyte is not absolutely required for its translation. Furthermore, sufficient Osk is present at this noncortical site that Vasa, whose localization is dependent upon Osk (Hay et al., 1990; Lasko and Ashburner, 1990) can be recruited (Fig. 2E-G).

\section{Molecular characterization of the enc locus}

The original encore allele, $e n c^{B B}$, was isolated as a P-element insertion mutation, BB129. The P element associated with this allele was used to isolate flanking genomic DNA by plasmid rescue. Subsequent isolation of overlapping genomic clones and restriction analysis produced a map of the genomic sequence surrounding the insertion site (Fig. 3A), and the entire genomic region has been subsequently sequenced. Using a $9 \mathrm{~kb}$ EcoRI fragment downstream of the insertion, several partial cDNAs were isolated from ovarian poly $(\mathrm{dT})$-primed libraries, and the longest of these (cDNA 4-1, 5.6 kb) was sequenced. Further screening of a randomly primed cDNA library and 5' RACE were used to obtain an additional $1.2 \mathrm{~kb}$ of enc transcript sequence. Primer extension analysis reveals a reverse-transcriptase stop site in the RNA at the site corresponding to the $5^{\prime}$ end of our RACE product, and another approximately $400 \mathrm{bp}$ further upstream (Fig. 3B), though we cannot be certain that this represents a true transcription start site. Alignment of the cDNA and genomic sequences reveals twelve introns, ranging from $56 \mathrm{bp}$ to $10.5 \mathrm{~kb}$, with the $10^{\text {th }}$ intron being unusual in that it possesses non-consensus $5^{\prime}$ and $3^{\prime}$ splice sites.

The cDNAs isolated from the poly(dT)-primed library fell into two main classes, which differed in the length of their $3^{\prime}$ UTRs. Using a probe common to both classes of cDNAs in northern analysis, transcripts of 7 and $8 \mathrm{~kb}$ were detected in wild-type ovarian RNA (Fig. 3C). Using a probe unique to the longer class of cDNAs, only the $8 \mathrm{~kb}$ transcript is detected (data not shown), strongly suggesting that the two transcripts differ only in the length of their $3^{\prime}$ UTRs. These transcripts are not detected in the $e n c^{B B}$ mutant using a probe that lies downstream of the BB129 P-element insertion (Fig. 3C). A truncated transcript is observed in this mutant when a probe upstream of the insertion is used (data not shown). The enc cDNA sequence is in total 6858nt, with a $3^{\prime}$ end corresponding to the $8 \mathrm{~kb}$ transcript, and hence falls short of the expected size. As primer extension analysis suggests that the $5^{\prime}$ end may lie approximately $400 \mathrm{nt}$ further upstream, bringing the total transcript size to only $7.2 \mathrm{~kb}$, a discrepancy still exists between our cDNA and the observed transcript size. This may arise from an aberrant migration of the RNA in northern analysis, from undetected splicing events, or from an inability to isolate the true $5^{\prime}$ end of the gene. We have also examined, by northern analysis, the expression of the neighboring gene $\operatorname{scs} \alpha$, encoding the alpha subunit of succinyl-CoA synthetase, and find its expression to be unaltered in $e n c^{B B}$ (data not shown).

\section{Transgenic rescue of enc}

enc RNA expression is disrupted in several enc alleles, as shown by northern analysis (above) as well as by in situ hybridization (data not shown), strongly suggesting that this transcription unit corresponds to the enc locus. In order to confirm this, we constructed an $e n c^{+}$transgene and tested its ability to rescue the enc mutant phenotype. A partial enc cDNA, pEnc5.5 (Fig. 3A), was placed under the control of the hsp83 promoter in a P-element transformation vector and several transformant lines were established. This transgene, expressed ubiquitously at $25^{\circ} \mathrm{C}$, was able to rescue the germline mitotic defect of enc females (Fig. 4). We also examined the ability of this transgene to rescue the coldsensitive ventralized egg defect of enc (Table 2) and observed a partial rescue of this phenotype. The failure to fully rescue the DV patterning defect is likely due to insufficient expression from this transgene at the lower temperatures at which this phenotype is penetrant. 
Table 2. Transgenic rescue of the enc ventralized egg phenotype

\begin{tabular}{|c|c|c|c|c|}
\hline & \multicolumn{3}{|c|}{ Egg shell phenotype (\%) } & \multirow{2}{*}{$\begin{array}{c}\text { Hatch rate } \\
(\%)\end{array}$} \\
\hline & I & II & III & \\
\hline enc $c^{U U 3} /$ enc $^{U U 3}$ & 13.9 & 73.9 & 12.2 & 26.8 \\
\hline $\mathrm{P}[\mathrm{enc}+] ;$ enc $c^{U U 3} / e^{-n c^{U U 3}}$ & 76.5 & 22.8 & 0.7 & 75.3 \\
\hline
\end{tabular}

The egg shell phenotype of enc mutant females was compared to mutants carrying multiple copies of the hspEnc5.5 transgene. Class I: two dorsal appendages. Class II: a single dorsal appendage. Class III: little or no dorsal appendage material. The majority of eggs appeared to be unfertilized ( $85 \%$ for $e n c^{U U 3}, 67 \%$ for the transgenic females), and hatch rates were derived from fertilized eggs only. Females were raised at room temperature, instead of the more non-permissive $18^{\circ} \mathrm{C}$, in order to increase expression from the transgene. Over 500 eggs of each genotype were scored.

\section{enc encodes a large protein with a highly conserved domain}

Though primer extension analysis indicates that we have not isolated the 5'end of the enc transcript, analysis of the cDNA sequence strongly suggests that we have isolated the translational start site. The $5^{\prime}$-most exon of enc contains multiple stop codons in all frames, and the second and third exons each begin with potential initiator methionine codons, in-frame and separated by $426 \mathrm{bp}$. The second methionine, unlike the first, is preceded by a consensus translational start sequence (Cavener, 1987), suggesting that the second methionine may be used for translational initiation. Furthermore, the transgene hspEnc5.5, which excludes the first methionine, can rescue the enc phenotypes. Thus while it is possible that both sites are used for translational initiation, the first appears dispensable for function. Hence all references to amino acid number assume the latter as the translational start site, corresponding to nucleotide 684 of the enc cDNA sequence, predicting an open reading frame of 1548 amino acids (Fig. 5A). There are no motifs in the protein sequence indicative of a particular biochemical function. Interestingly, however, database searches reveal homology to a group of closely related human cDNAs of unknown function that are over $40 \%$ identical with each other and most likely represent paralogous genes. The most similar of these to enc is KIAA0029 (GenBank accession Q15032). While the overall simlarity is low, a highly conserved domain within the Nterminal region is apparent: over 157aa, (aa 273-429 of Enc) these proteins are $47 \%$ identical and $67 \%$ similar (Fig. 5B). This domain is also present in a $C$. elegans predicted open reading frame (AAF39995). Thus enc encodes a large protein of unknown function with at least one domain that has been highly conserved.

\section{Enc protein is cytoplasmic and colocalizes with grk RNA}

The phenotypes of enc suggest roles in both cytoplasmic and nuclear processes, and we were thus interested in determining the subcellular distribution of Enc. Rat and rabbit polyclonal antibodies were raised against amino acids 132-339 of the Enc protein, and immunoprecipitation and western analyses of ovarian extracts show a $230 \mathrm{kDa}$ protein that is absent from $e n c^{B B}$ (Fig. 6A). This observed molecular mass is much higher than that predicted for Enc (approx. $170 \mathrm{kDa}$ if the second methionine is used for translational initiation, or approx. $185 \mathrm{kDa}$ if the first methionine is used) and the cause of this difference is unknown. Antibody stainings of wild-type ovaries show Enc to be a cytoplasmic protein. Under a variety of fixation conditions, Enc is not detected in nuclei, indicating that Enc's role in karyosome formation is likely indirect.

The mitotic phenotype of enc mutants suggests that enc acts to restrict cystocyte division. Thus it seemed reasonable that Enc expression within the germarium might coincide with exit from the mitotic cycle, at the formation of the 16-cell cyst. However, we detect Enc in all germline cells of the germarium (Fig. 6B), including the stem cells and dividing cystocytes. Thus Enc expression alone cannot inhibit cystocyte division. Enc begins to accumulate preferentially within the future oocyte shortly after formation of the 16-cell cyst. In midoogenesis, Enc can be seen transiently at the posterior edge of the oocyte, but by stage 9 assumes an anterior localization, and appears to be more concentrated at the dorsal side of the oocyte, above the oocyte nucleus (Fig. 6C). This pattern of localization is similar to that seen for $g r k$ RNA and protein, though Enc is not as tightly restricted to the dorsal side. In a double labeling experiment, Enc protein is seen to colocalize with grk RNA at the dorsal-anterior corner of the oocyte (Fig. $6 \mathrm{D}, \mathrm{E})$. While the localization of Enc protein around the oocyte nucleus is not dependent upon the presence of $g r k$ mRNA (data not shown), the localization of grk mRNA is perturbed in enc mutants, such that the RNA is less tightly restricted to the dorsal side of the oocyte (Hawkins et al., 1997). Since Enc does
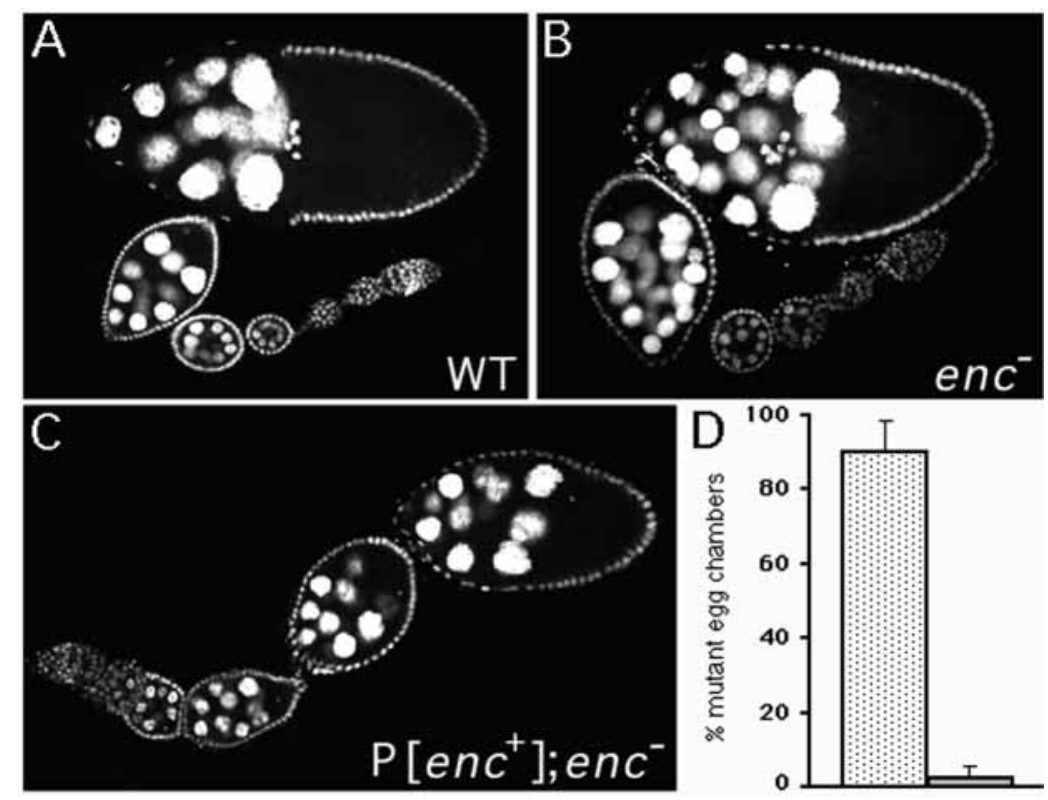

Fig. 4. Transformation rescue of the enc mitotic defect. (A) A wild-type ovariole, stained with OliGreen. Egg chambers contain 15 nurse cells and an oocyte. (B) An enc mutant ovariole; egg chambers contain 31 nurse cells and an oocyte. (C) Ovariole from an enc mutant carrying a transgene expressing a partial enc cDNA. Egg chambers contain 15 nurse cells and an oocyte. (D) A comparison of the frequency of egg chambers that have undergone an extra germline division in sibling enc ${ }^{U U 3}$ females without (stippled) and with (shaded) the enc transgene. Females were raised at $25^{\circ} \mathrm{C}$ and dissected 2 days after eclosion. 


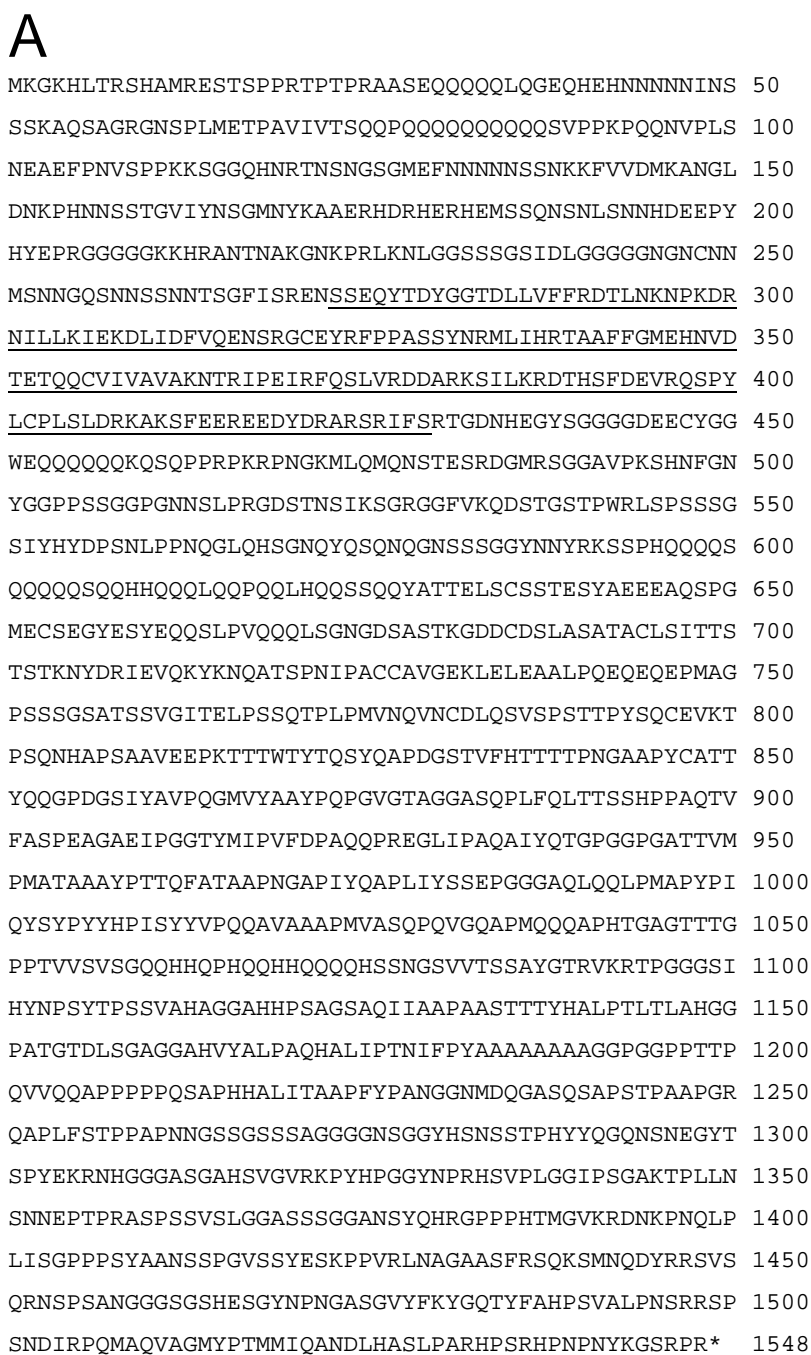

B

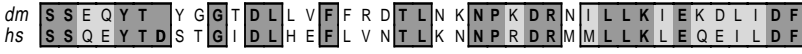

$d m$ VQ

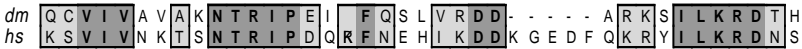

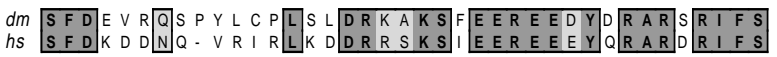

Fig. 5. (A) The Enc protein sequence. Enc bears homology to a group of human cDNAs, and the region of Enc that shows highest similarity to these is underlined. This domain of Enc is shown aligned with the human cDNA K1AA0029 ( $d m$, $h s$ respectively) (B). Over 157 amino acids, these proteins are $47 \%$ identical (dark gray) and $67 \%$ similar (light gray).

not contain known RNA-binding motifs, it is unlikely that it binds directly to the grk mRNA, though this possibility cannot be ruled out. It is possible that Enc is part of a complex of proteins required for the localization and translation of grk, or it could act indirectly, affecting the activity of a factor involved in these processes.

Within the male germline, four rounds of mitosis occur to generate 16 primary spermatocytes in a manner similar to that seen in female cystocyte division. We were thus interested in whether enc might also be expressed in the dividing cystocytes of males. As shown in Fig. 6F, Enc is expressed at the tip of the testis, where germline mitosis occurs. The possible function of enc in spermatogenesis has not been investigated, but male fertility does not appear to be affected in enc mutants.

\section{The enc ${ }^{R 17}$ mutation uncouples the early and late requirements for enc}

enc ${ }^{R 17}$ homozygous mutant females do not show the extra round of germline mitosis seen in all other enc mutants raised at $25^{\circ} \mathrm{C}$. However, this allele causes the cold-sensitive ventralized egg phenotype (Hawkins et al., 1997) as well as severe defects in karyosome formation. The enc ${ }^{R 17}$ allele was generated by excision of the A309 P element, which is inserted in the $5^{\prime}$ UTR of enc (Fig. 1). PCR analysis of enc ${ }^{R 17}$ genomic DNA shows that the excision removes sequences upstream of the original insertion, leaving downstream sequences intact (data not shown). Thus a likely explanation for the unique phenotype of $e n c^{R 17}$ is that enc expression is affected, in such a way that allows it to function in germline mitosis but not in the regulation of Grk levels. To address this possiblity, we examined Enc expression in $e n c^{R 17}$, and in several other enc mutants (Fig. 7). In wild type, Enc is expressed within the germarium and is detected within the oocyte throughout oogenesis (Fig. 7A). In mutants with enc alleles that affect both germline mitosis and DV patterning, Enc protein expression is affected at all stages (Fig. 7B,C). However, in enc ${ }^{R 17}$ mutant ovaries, Enc protein is detectable within the germarium and the early stages of oogenesis, but expression decreases and is undetectable by mid-oogenesis (Fig. 7D). These results are consistent with the patterns of enc RNA expression within these mutants (data not shown). Thus it appears that the enc $c^{R 17}$ allele represents a regulatory mutation that allows early expression of enc and hence does not display the mitotic defects characteristic of all other enc alleles.

\section{DISCUSSION}

\section{A role for enc in grk translation}

Mutants that exhibit reduced Gurken protein levels and abnormal oocyte nuclear morphology can be divided into at least two groups (Ghabrial and Schüpbach, 1999). Mutations in the first class cause activation of a meiotic checkpoint which in turn represses Grk translation and prevents karyosome formation within the oocyte. The second class of mutations represent genes that act downstream of the meiotic checkpoint, such as vasa, to regulate Grk translation and karyosome formation. As enc mutations cause defects in both karyosome formation and Grk accumulation, we ordered enc function with respect to the $m e i-41$ checkpoint by analyzing the phenotype of females mutant for both enc and the meiotic checkpoint gene $m e i-41$. We found that, unlike okra and spn-B, the karyosome and Grk translation defects of enc mutants are not suppressed by a mutation in $m e i-41$, and hence these phenotypes in enc mutants are not caused by the activation of the mei-41 checkpoint.

Mutations in vasa (vas), like enc, cause defects in karyosome formation and Grk expression and are not 


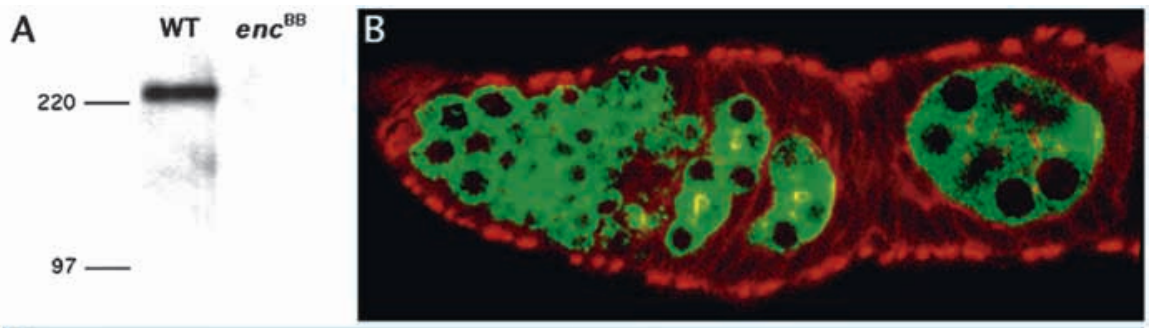

Fig. 6. Enc expression. (A) Enc immunoprecipitation-western blot showing a $230 \mathrm{kDa}$ band that is undetectable $e n c^{B B}$. (B) A wild-type germarium showing Enc expression (green) in all germline cells; actin is highlighted in red. (C) A wild-type ovariole, showing Enc accumulation within the oocyte, and subsequent localization within the oocyte to the anterior, with a higher concentration at the dorsal side. (D,E) A doubly labeled stage 9 egg chamber showing Enc protein (D) accumulating above the oocyte nucleus, and (E) in situ hybridization to grk RNA. (F) Enc expression is seen at the tip of a wild-type testis, where germline cell divisions occur.
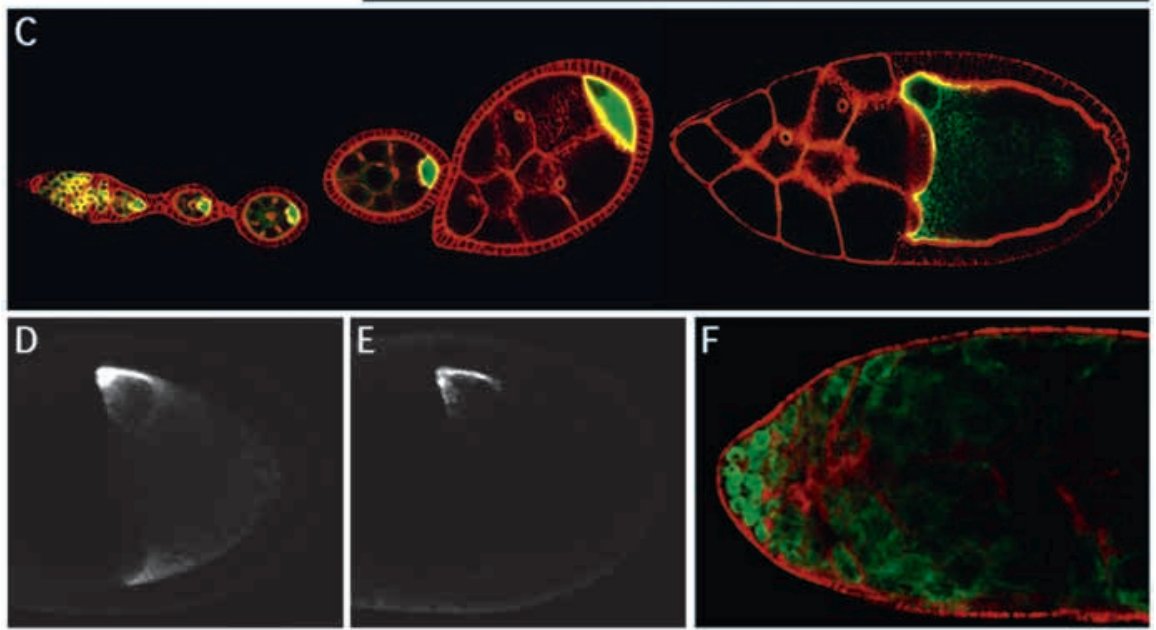

suppressed by mei-41 (Styhler et al., 1998; Tomancak et al., 1998; Ghabrial and Schüpbach, 1999). A potential translational regulator of $g r k$, Vas appears to be modified in response to activation of the meiotic checkpoint, as indicated by its altered migration in western analysis (Ghabrial and Schüpbach, 1999). However, the factors that mediate this modification are unknown, as is the mechanism by which meiotic progression is relayed to the cytoplasm. The similarities between the vas and enc phenotypes make enc a candidate for such a factor. We reasoned that if enc was required for Vas activity, and was in turn inhibited by the activation of the checkpoint, then the modification of Vas in enc mutant extracts would be altered from wild type. However, Vas expression appears normal in enc mutant ovaries, both by western analysis and by antibody staining, and thus enc function is unlikely to lie upstream of vas. Enc therefore affects karyosome formation and Grk protein accumulation either independently of the mei-41/vas pathway or functions downstream of vas to affect these processes.

\section{Oskar expression in enc}

Another gene implicated in both Grk translation is aubergine (aub), originally identified by mutations that caused production of ventralized eggs (Schüpbach and Wieschaus, 1991). As aub mutants also display abnormal karyosome morphology (Ghabrial et al., 1998), it appears that $a u b$ and enc may be participating in the same processes. Studies of aub have revealed an additional role in posterior body patterning, and it was found that aub egg chambers showed defects in both Gurken and Oskar translation (Wilson et al., 1996). We were thus interested in determining whether enc mutants also display defects in Osk accumulation. We found that Osk expression is still detected in enc mutant egg chambers: Osk protein is seen at the posterior pole at a frequency similar to that of the localization of osk RNA to the posterior. Thus enc, unlike $a u b$, does not appear to be required for Osk translation.

Mutations that affect AP patterning, such as those in enc and grk, frequently cause a mislocalization of osk mRNA, which is normally found at the posterior pole, to the middle of the oocyte. Previous analysis of mutations affecting the localization of $o s k$, including mutations in $g r k$, indicated that in this situation osk mRNA is not translated (Markussen et al., 1995). It appeared from these studies that localization of $o s k$ mRNA to the posterior cortex of the ooctye is required for its
Fig. 7. Enc expression in enc mutants. In a wild-type ovariole, Enc protein is expressed within the germarium and localizes to the oocyte of early stage egg chambers. $e n c^{U U 3}$ and $e n c^{A 309}$, like most enc alleles, show reduced Enc expression at all stages. $e n c^{R 17}$, unlike other enc alleles, shows Enc expression early in oogenesis and is not associated with a mitotic defect. However, Enc accumulation is severely affected in more mature egg chambers (arrow) and is undetectable by stage 9 (not shown).
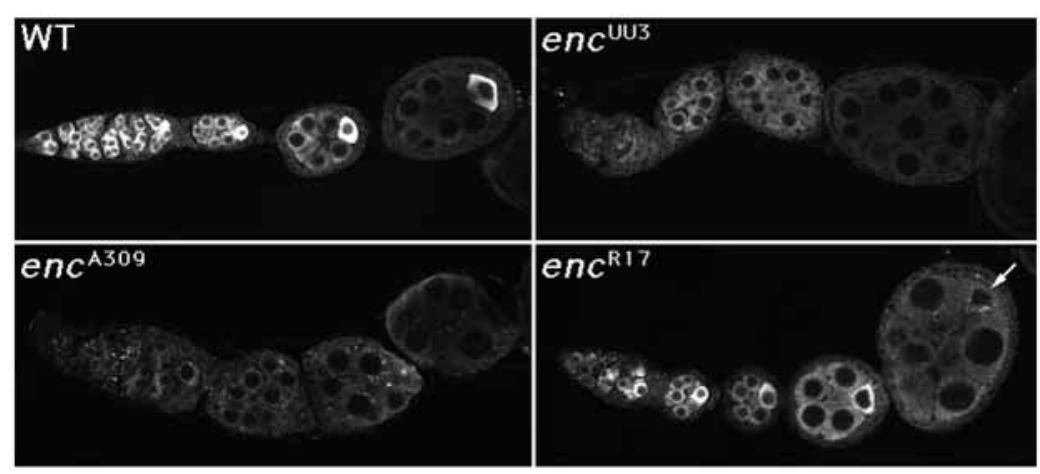
translation. However, in our analysis of Osk expression in enc mutant egg chambers, we find that mislocalized osk is capable of being translated, and hence cortical localization is not absolutely required for translation. We have found that, in addition, osk RNA is more tightly localized to the center of the oocyte in enc than in grk mutants, though the reason for this difference is not clear. One possible explanation is raised by the fact that Osk protein is required for the maintenance of its own RNA at the posterior of the oocyte (Rongo et al., 1995): osk RNA might be more tightly localized to the center of enc oocytes as a consequence of its translation. This situation might arise if, in enc but not in grk mutants, factors required for derepression of osk translation, such as p68 (Gunkel et al., 1998), were active in the center of the oocyte. However, we cannot at present distinguish whether the discreteness of osk mRNA mislocalization in enc mutants is a cause or a consequence of its translation.

\section{Two temporally distinct requirements for enc function}

The mitotic phenotype and the ventralized egg phenotype produced by mutations in enc have been shown to be inversely affected by temperature: the extra division phenotype is sensitive to increased temperature (ts), whereas the defect in Grk accumulation is cold-sensitive (cs) (Hawkins et al., 1996; 1997). While the ts and cs phenotypes of enc are independent, it remained a possibility that enc is required only once, early in oogenesis, to function in multiple processes. However, we found that enc functions at least twice during oogenesis. Mutations in enc that eliminate Enc expression from all stages produce both defects, while the unique allele $e n c^{R 17}$, in which Enc protein is detected in early but not later stages, shows no mitotic phenotype but does display severe patterning defects. While the levels of Enc expressed at these early stages are lower than in wild type, the separation of phenotypes in this mutant cannot be explained by different threshold requirements for Enc at a single stage of development. If this were true, the patterning defects in $e n c^{R 17}$ would be weaker than from alleles producing severe mitotic defects. This, however, is not the case. Thus the requirement for enc in Grk expression is temporally distinct from its role in germline mitosis.

\section{encore in the regulation of germline mitosis}

Mutations in enc cause one extra round of mitosis in the germline, and thus the wild-type function of enc is most likely to restrict germline division. Previous analysis has indicated that this may occur through regulation of the expression of the gene bag of marbles (bam). bam is implicated in the regulation of germline division, and its expression in wild-type germaria is consistent with a role in promoting cystocyte division. bam RNA is expressed in cystoblasts and two-cell cysts (McKearin and Spradling, 1990), and it has been proposed that Bam is a limiting factor in the biogenesis of the fusome (Ohlstein and McKearin, 1997), a vesicle-rich structure that spans the dividing cyst and is implicated in the control of germline cell division (for review, see de Cuevas et al., 1997).

In enc mutants that show a high penetrance of the extra division phenotype, bam RNA expression is expanded. Importantly, this expansion appears to be required for the enc extra division, as a null mutation in bam acts as a dominant suppressor of the enc mitotic defect. Thus it appears that enc normally acts to restrict bam expression, either at the transcriptional level or by an effect on bam RNA stability. Antibody stainings reveal Enc to be a cytoplasmic protein, consistent with a role in the regulation of bam RNA stability but not excluding the possiblity of an indirect role in bam transcription. Furthermore, we find that the simplest model for Enc function, that its expression would be mutually exclusive with bam RNA expression, is not true: Enc is present in all germline cells including the dividing cystocytes. Thus, it is not the onset of Enc expression that limits cystocyte division. Rather, models for Enc function must account for the regulation of bam in a manner consistent with the continuous expression of Enc. For example, enc may limit the stability of the bam transcript such that it is found only in those cells in which it is transcribed. In this model, the transcription of bam would be controlled by another, spatially-restricted, factor.

Mutations in enc affect at least two RNA targets. We observe alterations in bam RNA expression, as well as grk RNA localization and, most likely, translation (although a role for enc in Grk protein stability rather than translation cannot be ruled out). A role for enc in the regulation of these RNAs is consistent with the localization of Enc protein. The sensitivity of the enc mutant phenotypes to temperature is also typical of RNA-dependent processes (for examples, see Staley and Guthrie, 1999; Schwer et al., 1998). However, enc does not contain known RNA-binding motifs, and we have not been able to detect direct binding of Enc to these RNAs in vitro. Thus the alterations in RNA regulation in enc mutants are likely mediated by an Enc-interacting protein or proteins, and hence the identification of factors with which Enc interacts will be critical to the understanding of Enc function.

We are grateful to Gail Waring and Peter Tolias for cDNA libraries, Anne Ephrussi for anti-Osk antibody, Paul Lasko and Ira Clark for anti-Vasa antibody and advice, and Scott Hawley for providing us with the $m e i-41^{D 3}$ allele. Many thanks to Amin Ghabrial for performing Vasa western analyses and to Joe Goodhouse for help with confocal microscopy. We thank Laura Nilson and Amin Ghabrial for critical reading of the manuscript, and Amanda Norvell for helpful discussion. This work was supported by the US Public Health Service Grant PO1 CA41086 and by the Howard Hughes Medical Institute.

\section{REFERENCES}

Cavener, D. R. (1987). Comparison of the consensus sequence flanking translational start sites in Drosophila and vertebrates. Nucl. Acid Res. 15, 1353-1361.

de Cuevas, M., Lilly, M. A. and Spradling, A. C. (1997). Germline cyst formation in Drosophila. Annu. Rev. Genet. 31, 405-428.

Deng, W.-M. and Ruohola-Baker, H. (2000). Laminin A is required for follicle cell-oocyte signaling that leads to establishment of the anteriorposterior axis in Drosophila. Curr. Biol. 10, 683-686.

Ghabrial, A., Ray, R. P. and Schüpbach, T. (1998). okra and spindle-B encode components of the RAD52 DNA repair pathway and affect meiosis and patterning in Drosophila oogenesis. Genes Dev. 12, 2711-2723.

Ghabrial, A. and Schüpbach, T. (1999). Activation of a meiotic checkpoint regulates translation of Gurken during Drosophila oogenesis. Nat. Cell Biol. 1, 354-357.

González-Reyes, A., Elliott, H. and St. Johnston, D. (1995). Polarization of both major body axes in Drosophila by gurken-torpedo signalling. Nature 375, 654-658

González-Reyes, A., Elliott, H. and St. Johnston, D. (1997). Oocyte determination and the origin of polarity in Drosophila: the role of the spindle genes. Development 124, 4927-4937. 
Gunkel, N., Yano, T., Markussen, F.-H., Olsen, L.C. and Ephrussi, A. (1998). Localization-dependent translation requires a functional interaction between the $5^{\prime}$ and $3^{\prime}$ ends of oskar mRNA. Genes Dev. 12 , $1652-1664$

Hawkins, N. C., Thorpe, J. and Schüpbach, T. (1996). encore, a gene required for the regulation of germ line motisis and oocyte differentiation during Drosophila oogenesis. Development 122, 281-290.

Hawkins, N. C., Van Buskirk, C., Grossniklaus, U. and Schüpbach, T. (1997). Post-transcriptional regulation of gurken by encore is required for axis determination in Drosophila. Development 124, 4801-4810.

Hay, B., Jan., L. Y. and Jan, Y. N. (1988). A protein component of Drosophila polar granules is encoded by vasa and has extensive sequence similarity to ATP-dependent helicases. Cell 55, 577-587.

Hay, B., Jan, L. Y. and Jan, Y. N. (1990). Localization of vasa, a component of Drosophila polar granules, in maternal-effect mutants that alter embryonic polarity. Development 109, 425-433.

Kammermeyer, K. L. and Wadsworth, S. C. (1987). Expression of Drosophila epidermal growth factor receptor homolog in mitotic cell populations. Development 100, 201-210.

Kim, K. S., Febbraio, M., Han, T. H., Wessel, T. C., Park, D. H. and Joh, T. H. (1995). Analysis of gene expression by blotting techniques. In Gene Probes 2 (ed. B. D. Hames and S. J. Higgins), pp. 151-182. NY: Oxford University Press.

Lasko, P. F. and Ashburner, M. (1988). The product of the Drosophila gene vasa is very similar to eukaryotic initiation factor-4A. Nature 335, 611-617.

Lasko, P. F. and Ashburner, M. (1990). Posterior localization of vasa protein correlates with, but is not sufficient for, pole cell development. Genes Dev. 4, 905-921.

Markussen, F.-H., Michon, A.-M., Breitwieser, W. and Ephrussi, A. (1995). Translational control of oskar generates Short OSK, the isoform that induces pole plasm assembly. Development 121, 3723-3732.

McKearin, D. and Spradling, A. (1990). bag-of-marbles: a Drosophila gene required to initiate both male and female gametogenesis. Genes Dev. 4, 2242-2251.

Neuman-Silberberg, F. S. and Schüpbach, T. (1993). The Drosophila dorsoventral patterning gene gurken produces a dorsally localized RNA and encodes a TGF $\alpha$-like protein. Cell 75, 165-174.

Nilson, L. and Schüpbach, T. (1999). EGF receptor signaling in Drosophila oogenesis. Curr. Top. Dev. Biol. 44, 203-243.

Ohlstein, B. and McKearin, D. (1997). Ectopic expression of the Drosophila Bam protein eliminates oogeneic germline stem cells. Development 124, 3651-3662.

Pirrotta, V. (1986). Cloning Drosophila genes. In Drosphila, A Practical Approach (ed. D. B. Roberts), pp. 83-110. Oxford: IRL Press.

Roeder, G. S. (1997). Meiotic chromosomes: it takes two to tango. Genes Dev. 11, 2600-2621.

Rongo, C., Gavis, E. R. and Lehman, R. (1995). Localization of oskar RNA regulates oskar translation and requires Oskar protein. Development 121, 2737-2746

Roth, S., Neuman-Silberberg, F. S., Barcelo, G. and Schüpbach, T. (1995). cornichon and the EGF receptor signaling process are necessary for both anterior-posterior and dorsal-ventral pattern formation in Drosophila. Cell 81, 967-978.

Sambrook, J., Fritsch, E. and Maniatis, T. (1989). Molecular Cloning: A Laboratory Manual, 2nd ed. Cold Spring Harbor, NY: Cold Spring Harbor Laboratory Press.

Sapir, A., Schweitzer, R. and Shilo, B.-Z. (1998). Sequential activation of the EGF receptor pathway during Drosophila oogenesis establishes the dorsoventral axis. Development 125, 191-200.

Schüpbach, T. (1987). Germ line and soma cooperate during oogenesis to establish the dorsoventral pattern of egg shell and embryo in Drosophila melanogaster. Cell 49, 699-707.

Schüpbach, T. and Wieschaus, E. (1991). Female sterile mutations on the second chromosome of Drosophila melanogaster. II. Mutations blocking oogenesis or altering egg morphology. Genetics 129, 1119-1136.

Schwer, B., Linder, P. and Shuman, S. (1998). Effects of deletion mutations in the yeast Ces1 protein on cell growth and morphology and on high copy suppression of mutations in mRNA capping enzyme and translation initiation factor 4A. Nuc. Acids Res. 26, 803-809.

Spradling, A. C. (1993). Developmental genetics of oogenesis. In The development of Drosophila melanogaster (ed. M. Bate and A. MartinezArias), pp 1-70. NY: Cold Spring Harbor Laboratory Press.

Staley, J. P. and Guthrie, C. (1999). An RNA switch at the 5' splice site requires ATP and the DEAD box protein Prp28p. Mol. Cell 3, 55-64.

Styhler, S., Nakamura, A., Swan, A., Suter, B. and Lasko, P. (1998). vasa is required for Gurken accumulation in the oocyte, and is involved in oocyte differentiation and germline cyst development. Development 125, 15691578 .

Tautz, D. and Pfeifle, C. (1989). A non-radioactive in situ hybridization method for the localization of specific RNAs in Drosophila embryos reveals a translational control of the segmentation gene hunchback. Chromosoma 98, 81-85.

Tomancak, P., Guichet, A., Zavorsky, P. and Ephrussi, A. (1998). Oocyte polarity depends on regulation of gurken by Vasa. Development 125, 17231732.

Tomancak, P., Piano, F., Riechmann, V., Gunsalus, K. C., Kemphues, K. J. and Ephrussi, A. (2000). A Drosophila melanogaster homologue of Caenorhabditis elegans par-1 acts at an early step in embryonic-axis formation. Nat. Cell Biol. 2, 458-460.

Van Buskirk, C. and Schüpbach, T. (1999). Versatility in signalling: multiple responses to EGF receptor activation during Drosophila oogenesis. Trends Cell Biol. 9, 1-4.

Wilson, J. E., Connell, J. E. and Macdonald, P. M. (1996). aubergine enhances oskar translation in the Drosophila ovary. Development 122, 16311639 . 